\title{
A test of the hypothesis of an autopolyploid vs. allopolyploid origin for a tetraploid lineage: application to the genus Barbus (Cyprinidae)
}

\author{
A. CHENUIL*†‡, N. GALTIER $\dagger \S \&$ P. BERREBI† \\ †Laboratoire Génome et Populations, CNRS-UPR 9060, Université Montpellier II, 34095 Montpellier-cedex 05, \\ France; $\ddagger$ Institut des Sciences de l'Evolution de Montpellier, Université Montpellier II, 34095 Montpellier cedex 05, \\ France and §Laboratoire de Biométrie, Génétique et Biologie des Populations, CNRS-UMR 5558, \\ Université Claude Bernard Lyon 1, 43 bd du 11 Novembre 1918, 69622 Villeurbanne cedex, France
}

\begin{abstract}
A new method is described for determination of the origin of polyploid lineages. It tests the hypothesis that a tetraploid lineage originated via autopolyploidization vs. allopolyploidization. The method is based on the hypothesis that, in the case of autopolyploidy, any genetic marker in the first tetraploid ancestor is represented by two copies (one for each homoeologous chromosome of the haploid complement), whereas in allopolyploidy some markers absent from one of the hybridizing species will display one copy at most. The model requires knowledge of the phylogeny (topology and branch lengths) of a sample of species descending from the same tetraploidization event, together with the number of homoeologous copies present in each species for a set of neutral markers. The likelihood of a given proportion of the markers being present in both homoeologous chromosome pairs of the ancestral tetraploid is expressed as a function of the deletion rate of a marker. In the case of an autopolyploid origin, this proportion equals one. A likelihood-ratio test was carried out to test this hypothesis. The method was used to examine five microsatellite loci in eight species of Barbus (sensu lato). Assuming the validity of the hypotheses on phylogenetic relationships and evolutionary rates, the test rejects the possibility that European tetraploid barbs originated through autopolyploidy. This is the first test that can reject autopolyploidy, and it would appear particularly useful for phylogenetic studies in taxa where hybridization is known and where, consequently, undetected reticulate evolution may impair phylogenetic reconstruction.
\end{abstract}

Keywords: autopolyploidy, Barbus, likelihood-ratio test, microsatellites, phylogeny, reticulate evolution.

\section{Introduction}

Polyploidy is a very common phenomenon in plants. Although less well studied in animals, several polyploid taxa are known. There are no polyploid families, genera or even species groupings in insects (Lokki \& Saura, 1980) or amphibians and reptiles (Bogart, 1980). In fish, however, several ancient polyploid families or genera are made up of several sexually reproducing species (Schultz, 1980). In vertebrate evolution, polyploidy is considered to have enabled the evolution of more

*Correspondence: Anne Chenuil, Laboratoire Génome et Populations, CNRS-UPR 9060, Université Montpellier II, 34095 Montpellier-cedex 05, France. E-mail: chenuil@crit.univ-montp2.fr complex forms of life by giving the opportunity for new functions to evolve (Ohno, 1970). Nadeau \& Sankoff (1997) recently provided strong support for this view, estimating that after the two polyploidization events in the tetrapod lineage, nearly half the genes evolved new functions instead of losing their function, which is considerably more than previously thought. Although polyploidy is recognized as being widespread and of evolutionary importance, little is known about the origins of higher order polyploid taxa.

Distinction can be made between two kinds of eupolyploidy. Autopolyploids are formed of genomes of the same species (possibly the same individual), whereas allopolyploids contain genomes from distinct taxa. Allopolyploidy is therefore associated with 
hybridization. The establishment of whether the origin of a tetraploid lineage is allopolyploid or autopolyploid is of crucial importance for phylogenetic studies. If a lineage has arisen through the hybridization of two species, as in the case of allopolyploidy, phylogenetic relationships are not properly described by a tree but by a net, whose reconstruction is impossible with standard methods. Conventionally, two approaches are used to determine whether a lineage is auto- or allopolyploid. These are: (i) the cytological approach, involving observations of meiotic cells; and (ii) the formal genetic approach based on analyses of the proportions of marker alleles transmitted in the progeny of controlled crosses. After autopolyploidization, multisomic inheritance of each chromosome type is observed, but only for a certain time. Two (or three in the case of hexaploidy) chromosome pairs then become independent, with the re-establishment of disomic inheritance, avoiding meiotic problems which resulted from the formation of multivalents (Dewet, 1980). Cytology can no longer reveal an autopolyploid origin when disomic inheritance is complete. Furthermore, multivalents may be observable only at early stages of meiotic division (e.g. if chiasma are rare) and remain undetected because they have already dissociated at the stages generally observed (Jackson \& Casey, 1980). Conversely, multivalent formation in an allotetraploid cannot be rigorously excluded if the hybridizing species were closely related. The observation of meiotic cells is not therefore a suitable method for distinguishing between auto- and allopolyploidy. As an alternative to cytological approaches, analyses of data from crosses have been used to infer multisomic inheritance (Demarly, 1958; Jackson \& Casey, 1980) and, recently, a Bayesian procedure for testing hypotheses was developed (Olson, 1997). Olson's method could be used to test the null hypothesis of allopolyploidy, but requires that the assumption that allopolyploidy cannot lead to multisomic heredity should be true. Today, using cytological tools and allozyme data (or data from Mendelian markers), information on the origin of polyploidy is only available when multivalents are observed or when multisomic inheritance is established. In such cases, one can, at best, conclude that tetraploidy occurred either via autopolyploidization or via allopolyploidization involving very close species; there is no case in which allopolyploidy can be established and autopolyploidy unequivocally rejected.

A new method is described here for discriminating between the auto- and allopolyploidy hypotheses. It is based on the fact that all the genetic markers of a tetraploid lineage were present in two copies in the haploid complement of the ancestral genome in the case of autopolyploidy, but not in the case of allopolyploidy.
For a set of related tetraploid species, the mean number of copies per locus in the ancestral genome at many loci is estimated from the observed number of copies in extant species. Autopolyploidy is rejected when the proportion of loci which were ancestrally double is significantly different from one. The new method has been applied to microsatellite data of six tetraploid species of Barbus.

\section{The model}

A stochastic model was designed to represent the evolution of the number of copies of a set of neutral loci in the genomes of diverging tetraploid lineages. A common Poisson process of deletion is assumed for copies of any locus within any lineage. The mean number of copies in all loci in the ancestral tetraploid genome is estimated to test the autopolyploidy hypothesis.

Let $n$ be the number of tetraploid species examined. Assuming that homologous chromosomes are identical with regard to the presence or absence of a locus (i.e. presence-absence polymorphism is ignored), there may be two (one copy for each homoeologous chromosome, referred to below as the two-copy state), one (one-copy state) or zero (null state) copies of one locus in the haploid complement of a tetraploid genome. It is assumed that the number of genomic copies in $n$ extant species has been determined experimentally for $k$ loci. Data therefore consist of a species/character matrix containing the number of copies of a given locus in a given species.

$\mathrm{B}$ is taken as the most ancient tetraploid common ancestor of the species investigated (thus B is the

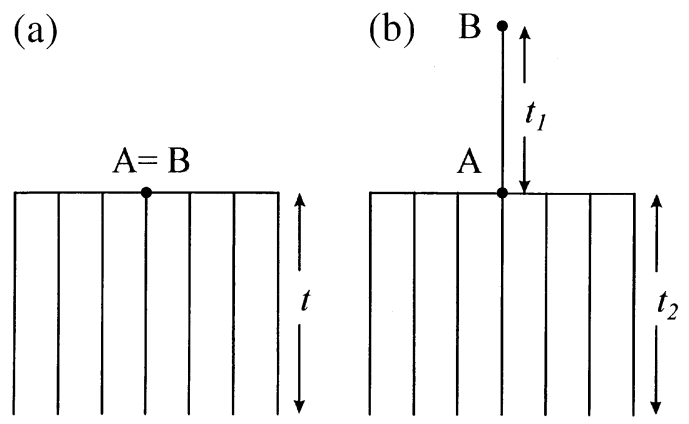

Fig. 1 (a) The comb model and (b) the rake model. B represents the first polyploid species; $\mathrm{A}$ is the most recent common ancestor of the surveyed species (confounded to B in the comb model). Branch lengths (in time units) are $t$ for the comb model, $t_{1}$ (ancestral branch) and $t_{2}$ (terminal branches) for the rake model. 
ancestral species which underwent tetraploidization, Fig. 1). It is assumed that the number of copies of a given locus did not increase after tetraploidization; extant loci therefore existed in the genome of B. Losses are allowed but not gains. Let $\phi$ be the proportion of two-copy loci within the ancestral genome (B), $1-\phi$ represents the frequency of one-copy loci in B. In the case of autotetraploidy, $\phi$ equals one. Let $\mu$ be the deletion rate of a given copy per time unit.

Given the phylogeny of the species investigated, and assuming that $\mu$ is constant in time, among loci and among lineages (molecular clock), one can compute the probability of a given column of the data matrix (i.e. the distribution of two-copy, one-copy and null states among $n$ species for a given locus) as a function of the parameters of the model, namely $\phi$ and $\mu$. The likelihood function for the whole data set is derived by multiplying these probabilities for $k$ loci, making it possible to estimate $\phi$ and to test the $\phi=1$ hypothesis using a likelihood-ratio test. Exact likelihood functions were derived in two particular cases, namely starphylogeny (referred to below as the comb model; Fig. 1a) and star-phylogeny with a root branch (the rake model; Fig. 1b). Maximum likelihood estimation of parameters was performed numerically using the simplex method (Press et al., 1992).

\section{The comb model}

An unresolved radiation of $n$ species immediately following tetraploidization is assumed. Let $t$ be the time since radiation. Assuming a common deletion Poisson process of parameter $\mu$, the probability $\pi$ of loss of a given ancestral copy in a given lineage during time $t$ is as follows for any locus:

$\pi=1 \quad \mathrm{e}^{\mu t}$.

Let us consider one locus, say locus $i(1 \leq i \leq k)$. In a star phylogeny, all $n$ species are equivalent and data are reduced to the number of two-copy species $\left(d_{\mathrm{i}}\right)$ and of one-copy species $\left(s_{i}\right)$ at locus $i\left(0<s_{i}+d_{i} \leq n\right)$. Let $b$ be the (unknown) number of copies of locus $i$ in the ancestral species B. The likelihood function at locus $i$ is:

$$
\begin{aligned}
& L_{i}^{\mathrm{C}}\left(\phi, \pi \mid s_{i}, d_{i}\right)=\operatorname{Pr}\left(s_{i}, d_{i}\right) \\
& =\operatorname{Pr}\left(s_{i}, d_{i} \mid b=1\right) \times \operatorname{Pr}(b=1)+ \\
& \operatorname{Pr}\left(s_{i}, d_{i} \mid b=2\right) \times \operatorname{Pr}(b=2) \\
& =\left(\begin{array}{ll}
1 & \phi
\end{array}\right) \operatorname{Pr}\left(s_{i}, d_{i} \mid b=1\right) \\
& +\phi \operatorname{Pr}\left(s_{i}, d_{i} \mid b=2\right),
\end{aligned}
$$

where superscript $\mathrm{C}$ is for the 'comb' model.

If at least one species has two copies, $\operatorname{Pr}\left(s_{i}, d_{i} \mid b=1\right)$ is zero because the number of copies is assumed not to increase after polyploidization. The likelihood at locus $i$ as a function of parameters $\phi$ and $\pi$ is therefore:

if $d_{i} \neq 0$,

$$
\begin{aligned}
& L_{i}^{\mathrm{C}}\left(\phi, \pi \mid s_{i}, d_{i}\right)=\phi\left(\begin{array}{c}
n \\
d_{i}
\end{array}\right)\left(\begin{array}{cc}
n & d_{i} \\
& s_{i}
\end{array}\right) \\
& \times 2^{s_{i}}(1 \quad \pi)^{2 d_{i}+s_{i}} \pi^{2 n} \quad 2 d_{i} s_{i} ;
\end{aligned}
$$

if $d_{i}=0$,

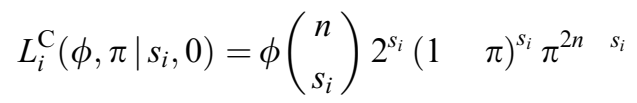

$$
\begin{aligned}
& +\left(\begin{array}{ll}
1 & \phi
\end{array}\right)\left(\begin{array}{l}
n \\
s_{i}
\end{array}\right)\left(\begin{array}{lll}
1 & \pi
\end{array}\right)^{s_{i}} \pi^{n} \quad s_{i} ;
\end{aligned}
$$

where combinatorial factors account for the many ways of drawing $d_{i}$ two-copy species and/or $s_{i}$ one-copy species among $n$ species and of drawing one out of two ancestral homologous copies in the cases of a single deletion from a double-copy ancestor.

The likelihood function for the whole data set is given by:

$L^{\mathrm{C}}(\phi, \pi)=\prod_{i=1}^{k} L_{i}^{\mathrm{C}}\left(\phi, \pi \mid s_{i}, d_{i}\right)$.

The 'comb' model is a two-parameter model.

\section{The rake model}

In this case, time $t_{1}$ elapsed between the tetraploidization event and radiation, which occurred $t_{2}$ time units ago. Let $\mathrm{A}$ be the most recent common ancestor of the $n$ species investigated. Let $\pi_{1}$ and $\pi_{2}$ be the probabilities of loss of a given copy in a given lineage during times $t_{1}$ and $t_{2}$, respectively:

$\pi_{1}=1 \quad \mathrm{e}^{\mu t_{1}}$ and

$\pi_{2}=1 \quad \mathrm{e}^{\mu t_{2}}$.

If $d_{i}$ and $s_{i}$ are the number of two-copy and one-copy species for locus $i$, respectively, and if $a$ is the number of copies of locus $i$ in ancestor $\mathrm{A}$, the likelihood function for locus $i$ is:

$$
\begin{aligned}
L_{i}^{\mathrm{R}}\left(\phi, \pi_{1}, \pi_{2} \mid s_{i}, d_{i}\right)= & \operatorname{Pr}\left(s_{i}, d_{i} \mid a=1\right) \times \operatorname{Pr}(a=1)+ \\
& \operatorname{Pr}\left(s_{i}, d_{i} \mid a=2\right) \times \operatorname{Pr}(a=2),
\end{aligned}
$$

where $\operatorname{Pr}\left(s_{i}, d_{i} \mid a=1\right)$ and $\operatorname{Pr}\left(s_{i}, d_{i} \mid a=2\right)$ are functions of $\pi_{2}$ (see the 'comb' model), and where $\operatorname{Pr}(a=1)$ and $\operatorname{Pr}(a=2)$ are functions of $\phi$ and $\pi_{1}$, namely:

$\operatorname{Pr}(a=1)=2 \phi \pi_{1}\left(\begin{array}{ll}1 & \pi_{1}\end{array}\right)+\left(\begin{array}{ll}1 & \phi\end{array}\right)\left(\begin{array}{ll}1 & \pi_{1}\end{array}\right)$ and 
$\operatorname{Pr}(a=2)=\phi\left(1 \quad \pi_{1}\right)^{2}$.

The likelihood at locus $i$ becomes:

if $d_{i} \neq 0$,

$$
\begin{aligned}
& L_{i}^{\mathrm{R}}\left(\phi, \pi_{1}, \pi_{2} \mid s_{i}, d_{i}\right)=\phi\left(\begin{array}{ll}
1 & \pi_{1}
\end{array}\right)^{2}\left(\begin{array}{c}
n \\
d_{i}
\end{array}\right)\left(\begin{array}{cc}
n & d_{i} \\
& s_{i}
\end{array}\right) \\
& \times 2^{s_{i}}\left(1 \quad \pi_{2}\right)^{2 d_{i}+s_{i}} \pi_{2}^{2 n} \quad 2 d_{i} \quad s_{i} ;
\end{aligned}
$$

if $d_{i}=0$,

$$
\begin{aligned}
& L_{i}^{\mathrm{R}}\left(\phi, \pi_{1}, \pi_{2} \mid s_{i}, 0\right)=\phi\left(\begin{array}{ll}
1 & \pi_{1}
\end{array}\right)^{2}\left(\begin{array}{c}
n \\
s_{i}
\end{array}\right) 2^{s_{i}}\left(\begin{array}{lll}
1 & \pi_{2}
\end{array}\right)^{s_{i}} \pi_{2}^{2 n} s_{i} \\
& +\left[2 \phi \pi_{1}\left(1 \quad \pi_{1}\right)+\left(\begin{array}{ll}
1 & \phi
\end{array}\right)\left(\begin{array}{ll}
1 & \pi_{1}
\end{array}\right)\right] \\
& \times\left(\begin{array}{c}
n \\
s_{i}
\end{array}\right)\left(\begin{array}{llll}
1 & \pi_{2}
\end{array}\right)^{s_{i}} \pi_{2}^{n} s_{i} .
\end{aligned}
$$

Again, the likelihood function for the whole data set is:

$L^{\mathrm{R}}\left(\phi, \pi_{1}, \pi_{2}\right)=\prod_{i=1}^{k} L_{i}^{\mathrm{R}}\left(\phi, \pi \mid s_{i}, d_{i}\right)$.

The 'rake' model is a three-parameter model. If external information on divergence times is available (e.g. an estimate of $t_{1} / t_{2}$ ), one parameter can be discarded.

\section{General case}

If the phylogenetic relationships among surveyed species are known, the likelihood function can be computed using the method of Felsenstein (1981). Branch lengths can theoretically be estimated from the data, together with the ancestral proportion of two-copy loci, $\phi$, and the mutation rate, $\mu$. However, if external information about branch lengths is available (possibly from a molecular phylogeny study), fixed branch length values can be used, considerably reducing the number of parameters to be estimated, which may be necessary when a moderate number of loci is examined.

\section{Likelihood-ratio test}

The autopolyploidy hypothesis may be tested using the likelihood ratio on any of the above models. Let $L$ be the maximum likelihood for one of the above models (say the comb model with two parameters). Let $L^{\prime}$ be the maximum likelihood for the comb model with the additional assumption $(\phi=1)$ expected in the autopolyploidy hypothesis (one parameter). Under this hypothesis $(\phi=1)$, twice the log likelihood ratio is $\chi^{2}$ distributed (1 degree of freedom):

2. $\log \left(L / L^{\prime}\right) \approx \chi_{1}^{2}$.

If $L$ is significantly higher than $L^{\prime}$, the autopolyploidy hypothesis should be rejected.

\section{Introduction on barb phylogeography}

The genus Barbus sensu lato (Cyprinidae) is a polyphyletic group with at least 800 species native to the Old World (Howes, 1987) and includes diploid, tetraploid and hexaploid lineages. As hybridization is not rare in Barbus (at least six pairs of B. barbus species (Mir, 1988; Machordom et al., 1990; Berrebi et al., 1993) are known to hybridize in nature), allopolyploidy may be a common phenomenon impairing phylogenetic reconstitution in this group. It is therefore important to determine whether particular polyploid groups have an allopolyploid origin.

Diploid species (48-52 chromosomes) are widespread from Asia to Africa. These small species are not well known and are probably polyphyletic. The only two groups of barbs considered to be monophyletic (Berrebi et al., 1996 and unpubl. data) are the subgenera Barbus (Euro-Mediterranean barbs), grouping all tetraploid species from Europe, western Asia and North Africa and Labeobarbus Pellegrin 1939, including species from throughout Africa and among which all the karyotyped species are hexaploid (Golubtsov \& Krysanov, 1993; Krysanov \& Golubtsov, 1996). Tetraploid species of barb not belonging to the subgenus Barbus are known in South Africa and in east and south Asia. Numerous other diploid and polyploid lineages exist but their phylogenetic relationships are far from established (Berrebi et al., 1996).

All Barbus are thought to be Asian in origin (Banarescu, 1973). The subgenus Barbus probably spread west by a 'Siberian' pathway, whereas the Labeobarbus subgenus probably followed an 'Ethiopian' pathway to colonize Africa (Darlington, 1948). These hexaploids probably did not originate from a tetraploid of the Siberian lineage, and may be derived from a deeper branch (Golubtsov \& Krysanov, 1993; C. Tsigenopoulos, pers. comm.).

Primer pairs for six microsatellite loci were isolated in two Barbus species in a previous study. For the present study, additional species were surveyed in order to obtain their copy number for extant microsatellite loci. Assuming that homoeologous copies of the loci were actually revealed (in contrast with tandem repeats for example), the test described above can be used to infer the origin of tetraploidy in Euro-Mediterranean barbs.

\section{Materials and methods}

Chenuil et al. (1997) established the allelic distributions of five of these loci for the two species $B$. (b.) barbus L., 1758 and $B$. (b.) meridionalis Risso, 1826, together with two hexaploid species, Barbus s.l., B. (Labeobarbus) intermedius Rüppel, 1837 and Varicorhinus beso Rüppel, 1837. Four further species of the subgenus Barbus were investigated in the study reported here, and also four 
other cyprinid and one cobitid species. The total sample thus comprised six tetraploid species of the subgenus Barbus (two species of the western European lineage, two species of the Iberian Peninsula lineage and two species of the North African lineage) and two African hexaploid species from Ethiopia, one belonging to the subgenus Labeobarbus and one still classified in a separate genus, Varicorhinus, although it is probably very close to Labeobarbus (Golubtsov \& Krysanov, 1993; Berrebi, 1995; Berrebi \& Valiushok, 1998).

Table 1 shows the loci, the corresponding primers and the standard PCR conditions used. For five loci, the genotypes were assessed of 414 individuals collected in the Lergue river (France). The sample included the following taxa: $B .(b$.$) meridionalis (36 individuals),$ B. (b.) barbus (28 individuals) and their natural hybrids (350 individuals). These taxa belong to the western lineage of European barbs (Berrebi, 1995). For the Iberian Peninsula lineage, four individuals of each species, B. (b.) bocagei Steindachner, 1865 and B. (b.) steindachneri Almaça, 1967, were caught in the Jerte river (Spain). For the North African lineage, four individuals per species were sampled, respectively, in the Draa river (Atlantic drainage) for $B$. (b.) pallaryi Pellegrin, 1919 and the Massa river (Mediterranean drainage) for B. (b.) massaensis Pellegrin, 1922 in Morocco. For the hexaploid species, 63 individuals of $B$. (l.) intermedius, and 15 individuals of Varicorhinus beso were collected in Lake Tana (Ethiopia). Other cyprinid genera included a tetraploid species, the carp (Cyprinus carpio L., 1758), several diploid species, the gudgeon (Gobio gobio L., 1758), the bream Abramis brama L., 1758), and the roach (Rutilus rutilus L., 1758), and a diploid species, the loach (Nemacheilus barbatulus L., 1766, Cobitidae). One individual of each of these species was caught in the Lergue river.
DNA was extracted by either: (i) a phenol extraction method from muscle or from liquid enzymatic extract (samples that had been used for allozymic studies); or (ii) boiling $5 \mu \mathrm{L}$ blood cells in $500 \mu \mathrm{L}$ of a solution containing 5\% of Chelex 100 (Bioprobe). Polymerase chain reaction amplification was carried out in $20 \mu \mathrm{L}$ with 5 pmol of each primer (the $F$ primer was $5^{\prime}$ labelled with fluorescein), 0.5 units $T f$ thermostable polymerase (Promega), $200 \mu \mathrm{M}$ each dNTP, and variable amounts of $\mathrm{MgCl}_{2}$ (3 mM for locus Barb79, $1.5 \mathrm{~mm}$ for other loci), in $T f l$ buffer. Polymerase chain reaction programmes consisted of $3 \mathrm{~min}$ at $92^{\circ} \mathrm{C}$ followed by 30 cycles $(35 \mathrm{~s}$ at $90^{\circ} \mathrm{C}, 35 \mathrm{~s}$ at $46-58^{\circ} \mathrm{C}$ according to the locus and $35 \mathrm{~s}$ at $72^{\circ} \mathrm{C}$ ) for all loci, except for loci Barb79 and Barb59 for which the extension step was extended $\left(50 \mathrm{~s}\right.$ at $\left.72^{\circ} \mathrm{C}\right)$. When testing for cross-specific amplification, PCR was performed systematically using programmes at lower annealing temperatures $\left(-6^{\circ} \mathrm{C}\right)$, with the usual (i.e. determined for B. barbus - B. meridionalis hybrids for each locus) and doubled $\mathrm{MgCl}_{2}$ concentrations to verify the absence of amplification product. However, in all cases when allospecific amplification was possible, it worked with the standard conditions.

A locus was determined as duplicate (either two disomic loci or one tetrasomic locus) when an individual displayed more than two alleles.

\section{Results}

\section{Microsatellite loci across Euro-Mediterranean Barbus species}

The results of allospecific amplification and expressed ploidy levels are recorded in Table 2. To summarize, five out of six loci are conserved between B. barbus and $B$. meridionalis, four of them are conserved between the

Table 1 Primer sequences, main repeat motif and standard PCR conditions for each locus

\begin{tabular}{|c|c|c|c|c|}
\hline Main motif & Locus name & Primers $\left(5^{\prime}-3^{\prime}\right)$ & $\begin{array}{c}\text { Annealing } \\
\text { temperature }\left({ }^{\circ} \mathrm{C}\right)\end{array}$ & $\begin{array}{l}\text { Extension step } \\
\text { (s) }\end{array}$ \\
\hline $\mathrm{CA}$ & Barb54 & $\begin{array}{l}\text { F54: GTTGTTTTGATTCACACTGAG } \\
\text { R54: TACCATCTGCTGCTGCTTC }\end{array}$ & 58 & 35 \\
\hline GATA & Barb59 & $\begin{array}{l}\text { F59: CTGTATCCATCACATAGGCT } \\
\text { R59: CATGATTTAATAGAACACACAC }\end{array}$ & 56 & 50 \\
\hline GATA & Barb65 & $\begin{array}{l}\text { F65: AATAATGCACTAAAACTGGGG } \\
\text { R65: CTTGTAATCAGACTACAGTTA }\end{array}$ & 58 & 35 \\
\hline GATA & Barb79 & $\begin{array}{l}\text { F79: GAGTGAATCATTACATCCCT } \\
\text { R79: GCTTTTCTTGTATTAGTATTT }\end{array}$ & 46 & 50 \\
\hline ATTT & Barb37 & $\begin{array}{l}\text { F37: AAATACGCTCTCCTCATTAC } \\
\text { R37: GTACAAAAGCAAAAATAAATTA }\end{array}$ & 50 & 35 \\
\hline ATTT & Barb62 & $\begin{array}{l}\text { F62: GGCACAAAAATGGATTCATATC } \\
\text { R62: GTACACGAGCATATGGACAA }\end{array}$ & 58 & 35 \\
\hline
\end{tabular}

(c) The Genetical Society of Great Britain, Heredity, 82, 373-380. 
Table 2 Results of allospecific amplification tests $\nmid$

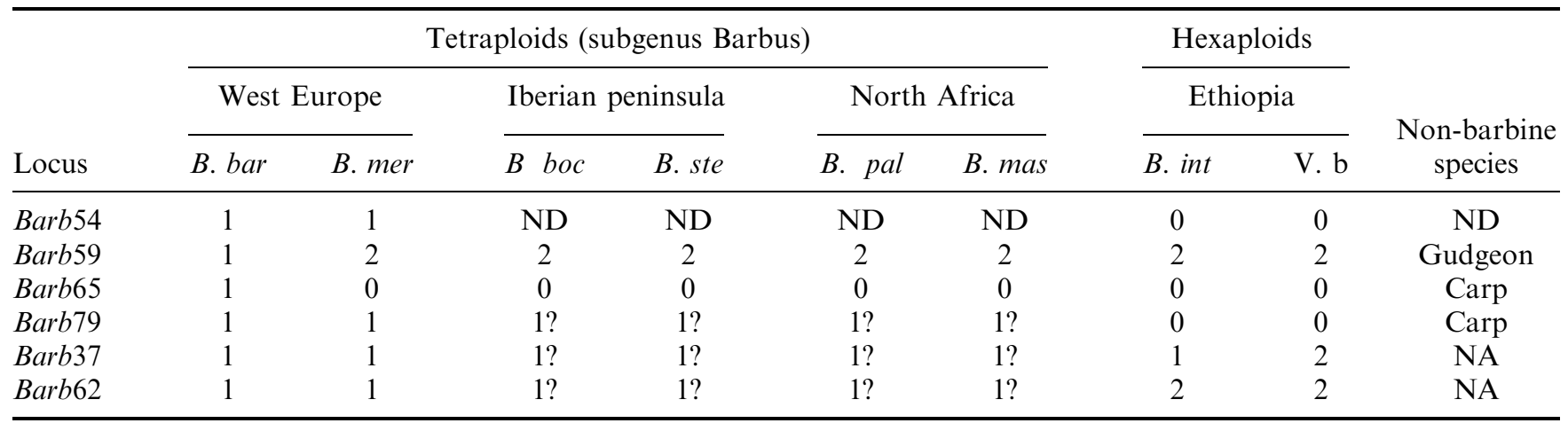

$\dagger$ Amplification patterns in B. (b.) barbus (B. bar), B. (b.) meridionalis (B. mer), B. (b.) bocagei (B. boc), B. (b.) steindachneri (B. ste), B. b.) massaensis (B. mas), B. (b.) pallaryi (B. pal), B. (l.) intermedius $(B$. int $)$ and Varicorhinus beso $(V . b)$, and in nonbarbine cyprinid or cobitid species. 0, amplification failure; 1, diploid expression (one or two different alleles per individual); 2, duplicate expression (up to four different alleles per individual); 1?, successful amplification producing one to two bands per individual, which was performed on too small a sample to establish unambiguously that the locus was single, although it is highly probable that this was the case (see text). ND, no data; NA, could not be amplified in nonbarbine species.

other four tetraploid Barbus species, and three of these loci amplify in both hexaploid barb species surveyed.

Barb37, Barb62 and Barb79 were considered to be simple loci in the six tetraploid Barbus species but their expressed ploidy could not be unambiguously established in the four species for which samples were too small. However, because for each locus more than four alleles were observed in the samples of the tetraploid species, and because no more than two bands were observed per individual, it is very likely that these loci are nonduplicate as will be assumed below.

Loci Barb79, Barb65, Barb37, Barb59 and Barb62 were found in species which diverged from the Barbus species investigated before tetraploidization: Barb59, Barb65 and Barb79 are present in a species which is unambiguously an outgroup of barbins (gudgeon) and the other loci are present in $V$. beso and $B$. (l.) intermedius which probably split before the tetraploidization event which led to our investigated species (C. Tsigenopoulos, pers. comm.). These five loci can therefore be used for our test.

A check was made (data not shown) that there was no presence-absence polymorphism for loci amplifiable in species $B$. barbus, $B$. meridionalis and $B$. intermedius, for which samples were large. This was confirmed by the analysis of progeny of crosses in B. meridionalis for the same loci (Chenuil et al., 1998).

Although microsatellites are, in general, selectively neutral, the priming sites may be embedded in coding regions; hence the present study checked the absence of codons in the flanking sequences of the microsatellite loci (Chenuil et al., 1997).

\section{Likelihood-ratio test of autopolyploidy}

Data consist of five loci whose copy number was determined in six species (Table 2). As the phylogenetic relationships among the surveyed species are unknown, the 'comb' and 'rake' models were used. With the 'comb' model, the likelihood-ratio test rejected the autopolyploidy hypothesis: twice the logarithm of the likelihood ratio was $8.26\left(P<0.01\right.$ for $\chi_{1}^{2}$ distribution $)$. When the three-parameter 'rake' model was assumed, numerical convergence was not reached, indicating that this data set does not retain enough information to estimate both ancestral state frequencies and branch lengths. However, providing (arbitrary) estimates of divergence times $t_{1}$ and $t_{2}$, maximum likelihood estimation of $\phi$ and $\mu$ became feasible and autopolyploidy was rejected $(P<5 \%)$ for a $t_{1} / t_{2}$ ratio lower than 0.4 . This value appears close to the ratio estimated for our barb species from cytochrome $b$ DNA sequences (C. Tsigenopoulos, pers. comm.).

\section{Discussion}

\section{Relevance of the model}

The stochastic evolutionary model used in this study is relevant for any set of neutral markers undergoing polarized evolution. This model is a special case of a three-state Markov process, where transition probabilities increasing the number of copies are assumed to be zero. Because the assumed process is not reversible, ancestral state frequencies (parameter $\phi$ ) can be estimated. Galtier \& Gouy (1998) used irreversible Markov models for molecular phylogeny, and found that accu- 
rate estimates of the frequencies of ancestral states can be obtained.

Note that neutrality is assumed for each locus, so that the probability of deletion of a given copy does not depend on the number of copies in the deleted genome. The model therefore seems poorly suited to allozyme loci, as natural selection probably maintains at least one copy per genome.

Several assumptions of this model are questionable. First, equal deletion rates among loci and among lineages are assumed. Both assumptions may be wrong in many cases. Unfortunately, although the relevant theory for addressing these questions has been developed (Wu \& Li, 1985; Yang, 1996), a large amount of data is required to check both hypotheses. Secondly, the method is sensitive to the underlying phylogeny. As an illustration, the autopolyploid hypothesis was rejected from our Barbus data with the 'comb' model, but the results were dependent on branch lengths when the 'rake' model was assumed. The power of the test should increase when information about phylogenetic relationships and branch lengths becomes available. Thirdly, ancestral presence-absence polymorphism is not taken into account. This assumption can be checked indirectly by examining polymorphism in extant species. If little or no polymorphism is found, one can be reasonably confident in assuming low ancestral polymorphism. Fourthly, the probability of gain of a copy is neglected. This is reasonable when the presence of a locus is assessed by PCR amplification (as in the present study) or by any method where sequence complementarity is involved (hybridization methods), because a mutation at any site may produce a loss, whereas a gain requires that the reverse mutation occurs at this site. Finally, a single locus duplication may be erroneously recorded as a homoeologous copy of a locus, if, as in our survey of tetraploid Barbus, loci are revealed by PCR amplification. However, this would leave the test conservative as allopolyploids, displaying additional copies, would be less easily distinguished from autopolyploids.

\section{Application to Barbus}

This application was performed using the comb and rake models although the phylogeny is currently unknown. The absence of published data on divergence times between the species examined prevented us from carrying out a more precise test on Euro-Mediterranean barbs, but this should be possible in the near future because promising molecular phylogenies using cytochrome $b$ sequences are being developed (C. Tsigenopoulos, pers. comm.).

The rejection of the autopolyploidy hypothesis is compatible with available data from independent studies which suggest that today the genome of Barbus is functionally diploid. First, cytological observations of hybrids $B$. (b.) barbus $-B$. (b.) meridionalis revealed no quadrivalents during meiosis (M. Castelli, pers. comm.); although in Salmonidae, another ancient tetraploid family, quadrivalents are commonly observed in males (Allendorf \& Thorgaard, 1984). Secondly, in the subgenus Barbus nearly all enzymatic loci have a strictly diploid expression, except for a very few loci where duplications independent of polyploidy might be involved (Berrebi et al., 1990, 1993).

\section{Potential applications of the test}

This test of the origin of polyploidy has a wide potential range of application. In Salmonids, for instance, it is currently accepted that tetraploidization occurred between 25 and $100 \mathrm{Ma}$, and that it was by autopolyploidization, because several quadrivalents are still observed (Allendorf \& Thorgaard, 1984). Application of the test reported here is feasible as microsatellite loci were isolated in this family by independent studies (e.g. Estoup et al., 1993; Slettan et al., 1993; Poteaux, 1995). Microsatellites have also been isolated in Carassius (Zheng et al., 1995), another well studied tetraploid genus of the Cyprinid family.

An alternative to microsatellite loci is the technique of in situ hybridization of fluorescent probes (FISH), which is the subject of rapid technical development (Lichter, 1997) and appears to be a promising technique for generating data for our test. This method has the advantage of directly visualizing the multiplicity of a locus and, moreover, the homology of the different copies, allowing the identification of tandem repeat copies of a locus.

\section{Acknowledgements}

We warmly thank Costas Tsigenopoulos, for providing important unpublished information, John Thompson, for careful reading and correction of an earlier version of the manuscript, François Bonhomme and Pierre Boursot, who read a recent version, I. Doadrio, A. Machordom, S. El Gharbi, L. Crespin and the Joint Ethiopian and Russian Biological Expedition for their participation in sampling. This work was supported by European Community Contracts EV5VCT920097 and CIPDCT925119.

\section{References}

ALLENDORF, F. W. AND THORGAARD, G. H. 1984. Tetraploidy and the evolution of salmonid fishes. In: Turner, B. J. (ed.) Evolutionary Genetics of Fishes, pp. 1-53. Plenum Press, New York. 
BANARESCU, P. 1973. Origin and affinities of the freshwater fish fauna of Europe. Ichthyologia, 5, 1-8.

BERREBI, P. 1995. Speciation of the genus Barbus in the north Mediterranean basin: recent advances from biochemical genetics. Biol. Cons., 72, 237-249.

BERREBI, P. AND VALIUSHOK, D. 1998. Genetic divergence among morphotypes of Lake Tana (Ethiopia) barbs. Biol. J. Linn. Soc., 64, 369-384.

BERREBI, P., LÉVÊQUe, C., CATTANEO-BERREBI, G., AGNÈSE, J.-F., GUÉGAN, J.-F. AND MACHORDOM, A. 1990. Diploid and tetraploid African Barbus (Osteichtyes, Cyprinidae): on the coding of differential gene expression. Aquat. Living Resour., 3, 313-323.

Berrebi, P., CATtANeO-BerRebi, G. AND LE BRUN, N. 1993. Natural hybridization of two species of tetraploid barbels: Barbus meridionalis and B. barbus (Osteichtyes, Cyprinidae) in southern France. Biol. J. Linn. Soc., 48, 319-333.

BERREBI, P., KOTTELAT, M., SKelton, P. AND RAB, P. 1996. Systematics of Barbus: state of the art and heuristic comments. Folia Zool., 45, 5-12.

BOGART, J. P. 1980. Evolutionary implications of polyploidy in amphibians and reptiles. In: Lewis, W. H. (ed.) Polyploidy: Biological Relevance, pp. 341-378. Plenum Press, New York. CHENuil, A., DeSMARAIS, E., POUYAUd, L. AND BerReBI, P. 1997. Does polyploidy lead to fewer and shorter microsatellites in Barbus (Teleostei: Cyprinidae)? Mol. Ecol., 6, 169-178.

CHENUIL, A., BINDA, O., CATTANEO-BERREBI, G., PONCIN, P. AND BERREBI, P. 1998. Relative reproductive success of males in polyandrous crosses of Barbus meridionalis revealed by microsatellite fingerprinting. Folia Zool., 47 (Suppl. 1), 5360.

DARLINGTON, P. J. 1948. The geographical distribution of coldblooded vertebrates. $Q$. Rev. Biol., 23, 105-123.

Demarly, y. 1958. Contribution à l'étude des tétraploides. $\mathrm{La}$ Semaine des Hopitaux, Ann. Génét., 34, 25-39.

DEWET, J. M. J. 1980. Origins of polyploids. In: Lewis, W. H. (ed.) Polyploidy: Biological Relevance, pp. 3-15. Plenum Press, New York.

ESTOUP, A., PRESA, P., KRIEG, F., VAIMAN, D. AND GUYOMARD, R. 1993. $(C T)_{n}$ and $(G T)_{n}$ microsatellites: a new class of genetic markers for Salmo trutta L. (brown trout). Heredity, 71, 488-496.

FELSENSTEIN, J. 1981. Evolutionary trees from DNA sequences: a maximum likelihood approach. J. Mol. Evol., 17, 368-376.

GALTIER, N. AND GOUY, M. 1998. Inferring pattern and process: maximum-likelihood implementation of a new, non-homogeneous model of DNA sequence evolution for phylogenetic analysis. Mol. Biol. Evol., 15, 871-879.

GOLUBTSOV, A. S. AND KRYSANOV, E. Y. 1993. Karyological study of some cyprinid species from Ethiopia. The ploidy differences between large and small Barbus of Africa. J. Fish Biol., 42, 445-455.
HOwES, G. J. 1987. The phylogenetic position of the Yugoslavian Cyprinid fish genus Aulopyge Heckel, 1841, with an appraisal of the genus Barbus Cuvier \& Cloquet, 1816, and the subfamily Cyprinidae. Bull. Br. Mus. Nat. Hist. (Zoology), 52, 165-196.

JACKSON, R. C. AND CASEY, J. 1980. Cytogenetics of polyploids. In: Lewis, W. H. (ed.) Polyploidy: Biological Relevance, pp. 18-44. Plenum Press, New York.

KRYSANOV, E. Y. AND GOLUBTSOV, A. S. 1996. Karyotypes of some Ethiopian Barbus and Varicorhinus from the Nile basin including Lake Tana morphotypes. Folia Zool., 45, $67-75$.

LICHTER, P. 1997. Multicolour FISHing: What's the catch? Trends Genet., 13, 475-478.

LOKKI, J. AND SAURA, A. 1980. Polyploidy in insect evolution. In: Lewis, W. H. (ed.) Polyploidy: Biological Relevance, pp. 277-312. Plenum Press, New York.

MACHORDOM, A., BERREBI, P. AND DOADRIO, I. 1990. Spanish barbel hybridization detected using enzymatic markers: Barbus meridionalis Risso Barbus haasi Mertens (Osteichthyes, Cyprinidae). Aquat. Living Resour., 3, 295-303.

MIR, S. 1988. A new natural intergeneric cyprinid hybrid from the Jordan River drainage, with a key to the large barbine cyprinids of the southern Levant. J. Fish Biol., 32, 931-936.

NADEAU, J. H. AND SANKOFF, D. 1997. Comparable rates of gene loss and functional divergence after genomic duplications early in vertebrate evolution. Genetics, 147, 1259-1266.

oHNo, s. 1970. Evolution by Gene Duplication. Springer, New York.

OLSON, M. 1997. Bayesian procedures for discriminating among hypotheses with discrete distributions: inheritance in the tetraploid Astilbe biternata. Genetics, 147, 1933-1942.

POTEAUX, C. 1995. Interactions génétiques entre formes sauvages et formes domestiques chez la truite commune (Salmo trutta fario L.). Ph.D. Thesis, University of Montpellier II.

PRESS, W. H., TEUKOLSKY, S. A., VETTERLING, W. T. AND FLANNERY, B. P. 1992. Numerical Recipes in C. Cambridge University Press, Cambridge.

SCHULTZ, R. J. 1980. Role of polyploidy in the evolution of fishes. In: Lewis, W. H. (ed.) Polyploidy: Biological Relevance, pp. 313-340. Plenum Press, New York.

SLETTAN, A., OLSAKER, I. AND LIE, O. 1993. Isolation and characterization of variable $(\mathrm{GT})_{\mathrm{n}}$ repetitive sequences from Atlantic salmon, Salmo salar L. Anim. Genet., 24, 195-197.

WU, C. I. AND LI, W.-H. 1985. Evidence for higher rates of nucleotide substitution in rodents than in man. Proc. Natl. Acad. Sci. U.S.A., 82, 1741-1745.

YANG, Z. 1996. Among-site variation and its impact on phylogenetic trees. Trends Ecol. Evol., 11, 367-372.

ZHENG, W., STACEY, N. E., COFFIN, J. AND STROBECK, C. 1995. Isolation and characterization of microsatellite loci in the goldfish Carassius auratus. Mol. Ecol., 7, 791-792. 